Results 1403 surveys were returned. Most respondents identified as bereaved family/friends and health or social care professionals. Respondents submitted a range of questions on services, communication, symptoms and perceptions of palliative care. 1331 respondents completed the second survey. In November 2014, 24 people participated in a workshop to prioritise the 'top 10' questions (using the Nominal Group Technique) which will be reported in January 2015.

Responses that are 'out of scope' of the JLA protocol will be analysed and reported separately.

Conclusion(s) The 'top 10' uncertainties will guide project partners' and other organisations' funding strategies, ensuring that future research is relevant to palliative care populations. We will discuss how the results of this project will guide the palliative and end of life care research agenda in future.

\title{
OP 003 RESEARCH PRIORITY SETTING IN PALLIATIVE AND END OF LIFE CARE: THE JAMES LIND ALLIANCE APPROACH CONSULTING PATIENTS, CARERS AND CLINICIANS
}

S Best, ${ }^{1}$ T Tate, ${ }^{1}$ B Noble, ${ }^{1}$ R Smith, ${ }_{1}^{1}$ J Tuft, ${ }^{1}$ N Tracey, ${ }_{1}^{1}$ A McCullugh, ${ }^{1}$ J Eley, ${ }^{2}$

J Black, ${ }^{3}$ M Stockton, ${ }^{4}$ A Cheesley, ${ }_{1}^{5}$ L Berry, ${ }_{1}^{5}$ C Burton, ${ }^{6}$ A Dechamps, ${ }^{7}$

E Stevens, ${ }^{8}$ A Penny, ${ }^{9} \mathrm{~L} \mathrm{McEnhill,}^{10}$ A McNair, ${ }^{11}$ B Cupid, ${ }^{12}$ B Maeso, ${ }^{13}$ D Chapman, ${ }^{14}$ S Mcllfatrick, ${ }^{15}$ A Nelson, ${ }^{16} \mathrm{~S}$ Sivell, ${ }^{16} \mathrm{~J}$ Baillie, ${ }^{16}$

J VanGodwin, ${ }^{16}$ B Candy, ${ }^{17} \mathrm{~N}$ Beaumont, ${ }^{18} \mathrm{~K}$ Cowan ${ }^{19}$. ${ }^{1}$ Marie Curie Cancer Care; ${ }^{2}$ NCRI Consumer Hub; ${ }^{3}$ National Council for Palliative Care; ${ }^{4}$ Association for Palliative Medicine of Great Britain and Ireland; ${ }^{5}$ Royal College of Nursing; ${ }^{6}$ Macmillan Cancer Support; ${ }^{7}$ St Christopher's Hospice; ${ }^{8}$ Scottish Partnership for Palliative Care; ${ }^{9}$ National Bereavement Alliance and Childhood Bereavement Network; ${ }^{10}$ Help the Hospices; ${ }^{11}$ Chief Scientist Office; ${ }^{12}$ Motor Neurone Disease Association; ${ }^{13}$ National Institute for Health Research; ${ }^{14}$ National Institute for Social Care and Health Research; ${ }^{15}$ All Ireland Institute of Hospice and Palliative Care; ${ }^{16}$ Marie Curie Palliative Care Research Centre, Cardiff University; ${ }^{17}$ Marie Curie Palliative Care Research Unit, University College London; ${ }^{18}$ Economic and Social Research Council; ${ }^{19}$ James Lind Alliance

\subsection{6/bmjspcare-2014-000838.3}

Introduction There can be a mismatch between what the public see as important unanswered questions and those which are actually researched. The Palliative and end of life care Priority Setting Partnership, facilitated by the James Lind Alliance (JLA), identified and prioritised questions about palliative and end of life care that people in the last years of life, current/ bereaved carers and professionals feel are important for research to address.

Aim(s) and method(s) A UK public survey (December 2013-April 2014) identified questions about support, care and treatment of people in the last years of life. Some 83 questions were formulated and prioritised via a second public survey. The resulting top 28 were prioritised in a workshop comprising patients, carers and clinicians to determine the top 10 research questions. 